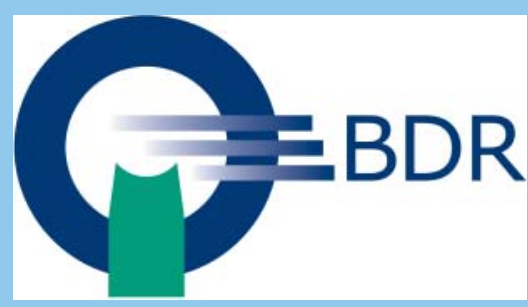

\title{
Interessante Buchempfehlungen für Radiologen
}

Weitere Rezensionen finden Sie auf unserer Webseite http://www.radiologenverband.de.

\section{Radiologische Diagnostik der Knochen und Gelenke}

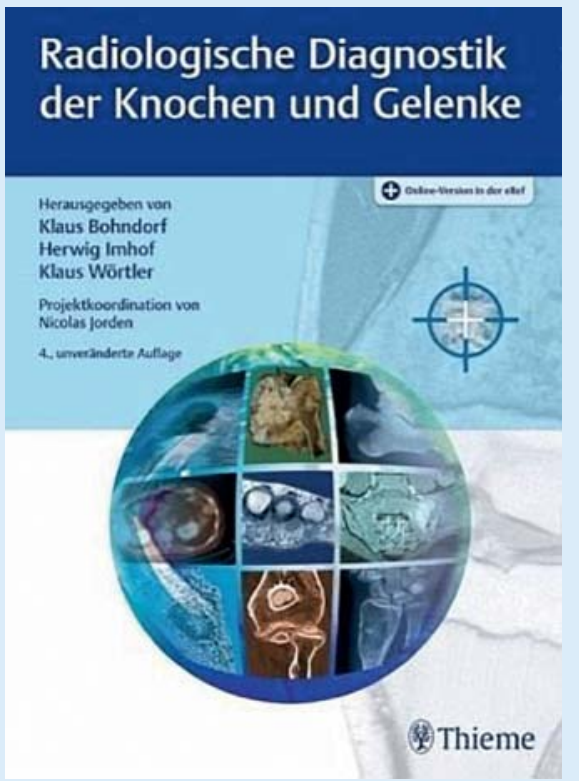

Klaus Bohndorf, Herwig Imhof, 543 Seiten, Georg Thieme Verlag, 4. unveränderte Auflage, 2017, ISBN-13: 978 - 3132414976 , $179,99 €$

Das Buch gliedert sich in insgesamt 13 Kapitel. Das große Thema „Trauma“ wird in zwei Kapiteln abgehandelt: im ersten werden die Grundlagen behandelt, im zweiten die Traumata der verschiedenen Körperregionen. Weitere Kapitel befassen sich mit Infektionen, Tumoren, Knochenmark, Nekrosen, Osteochondrosen, metabolisch/ hormonell/toxisch bedingten Osteopathien, Entwicklungsstörungen, rheumatischen Erkrankungen und Interventionen.
Des Weiteren gibt es ein Kapitel, das verschiedenes abhandelt, wie etwa M. Paget, Sarkoidose oder neurogene Osteoarthropathie. Das letzte Kapitel gibt einen kurzen Überblick über die Grundlagen der radiologischen Begutachtung des Skeletts und der Gelenke. In der hinteren Umschlaginnenseite gibt es noch einen Wegweiser zu wichtigen Klassifikationen und Einteilungen. Damit beinhaltet das Buch eine gute Grundlage dessen, was man als Facharzt für Radiologie zu diesem Thema wissen sollte. Es richtet sich im Wesentlichen an Ärzte in Weiterbildung, die mit diesem Buch einen guten Einstieg in die muskuloskelettale Radiologie erhalten. Es eignet sich zudem hervorragend für die Vorbereitung zur Facharztprüfung. Doch auch für Ärzte anderer Fachrichtungen, wie z. B. Orthopädie oder Unfallchirurgie, ist es ein wertvoller Wegweiser. Die einzelnen Kapitel sind einheitlich aufgebaut und bieten eine klare, übersichtliche Struktur. Bzgl. der anatomischen Grundlagen wird in der Regel auf die WebVersion des Buches verwiesen, was das Buch selbst kompakter und übersichtlicher hält. Die WebVersion erwirbt man zusammen mit dem Buch. Es hat einen Zugangscode auf der vorderen Umschlaginnenseite. In den einzelnen Kapiteln wird kurz auf die Pathophysiologie von Krankheitsbildern eingegangen sowie auf wichtige, zielführende Angaben zur Klinik des Patienten. Viel Raum nimmt, dem diagnostischen Stellenwert entsprechend, die Röntgendiagnostik ein. Sonografie, MRT und CTBefunde werden einzeln beschrieben, allerdings nur, sofern sie eine Relevanz für die Diagnostik besitzen. Hierzu finden sich stets die passenden Bildbeispiele. Auch der Beitrag der Nuklearmedizin findet entsprechende Berücksichtigung; leider finden sich hier jedoch nicht in allen Kapiteln passende Bild- beispiele. Die Stärken der jeweiligen Modalitäten und ihre Bedeutung für die betrachtete Erkrankung werden sehr gut herausgestellt. Die Abbildungen zeichnen sich durch hervorragende Qualität aus. Sie sind beschriftet und passen zum Text. Somit gibt es kein lästiges Vor- und Zurückblättern, um das passende Bild zum Text zu finden. Befunde sind im Bild markiert, was dem Anfänger die Zuordnung extrem erleichtert. Zudem gibt es Verweise zu Abbildungen, die zusätzlich in der WebVersion zu finden sind. Insgesamt hat das Buch über 2000 Abbildungen, davon 350 im Web. Dabei gibt es auch zahlreiche, didaktisch sehr gute Zeichnungen, bspw. für Fraktureinteilungen. Für Dinge, die man unbedingt wissen sollte und auch für Pitfalls gibt es die bei Thieme bekannten, blau unterlegten Kästchen ("Merke“ und „Cave“). Des Weiteren steht in der Rubrik „wichtig für den Befund“ das, was der Kliniker unbedingt wissen möchte, was also definitiv im Befundbericht nicht fehlen darf. Differenzialdiagnosen sind kurz erwähnt, ebenso die wichtigsten Unterscheidungsmerkmale. Mit €179,99 ist das Buch nicht günstig, aber m. E. aufgrund des hohen didaktischen Wertes und des herausragenden Bildmaterials sowie der integrierten WebVersion sein Geld wert. Ich habe bereits mit der 1. Auflage meinen Einstieg in das Thema gefunden und mich damit auch auf die Facharztprüfung vorbereitet. Die aktuelle Auflage ist umfangreicher und deutlich besser bebildert, sie hat dabei aber die klare Struktur und die sehr übersichtliche Gliederung behalten. Die WebVersion mit zusätzlichen Inhalten stellt einen weiteren Pluspunkt dar. Ich kann das Buch uneingeschränkt empfehlen.

Dr. med. Pia-Elisabeth Baqué, Mainz 


\section{Duale Reihe RADIOLOGIE}

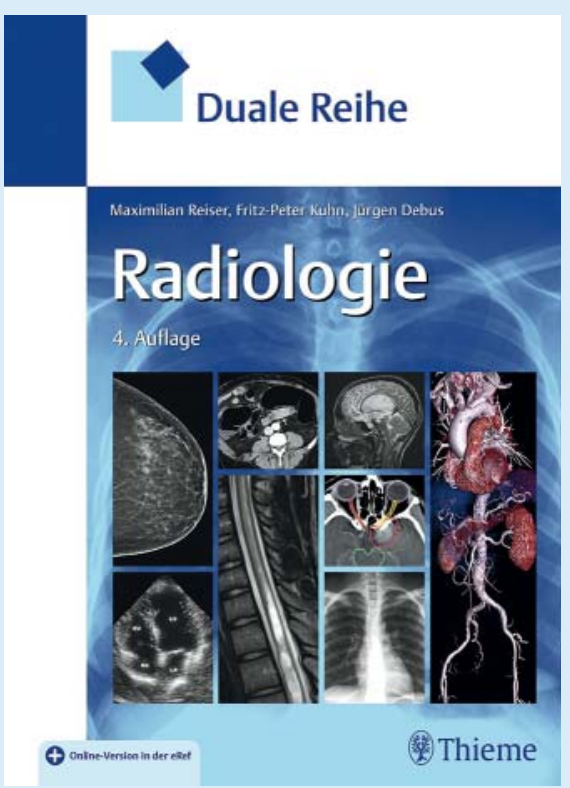

Maximilian Reiser, Fritz-Peter Kuhn, Jürgen Debus, Thieme, 2017, 4. Auflage als Buch im DINA-4-Format und mit online-Version (e-book) mit Zugangscode, 79,99€

Ende März 2017 erschien druckfrisch die 4. Auflage des Fachbuches RADIOLOGIE in der Dualen Reihe des ThiemeVerlags. Dieses mittlerweile unter Medizinstudenten und jungen Assistenzärzten sehr beliebte Fachbuch wurde seit dem Ersterscheinen im Jahre 2004 ständig den aktuellen Entwicklungen gemäß angepasst. Das weltweit verteilte Team von Radiologen um Prof. Reiser von der Uni München, Prof. Kuhn vom Klinikum Kassel und Prof. Debus von der Uni Heidelberg hat sich der Herausforderung gestellt auf knapp 800 Seiten ein (Kurz) Lehrbuch der gesamten Radiologie zusammenzustellen. Nun erscheint dieses Fachbuch, wie bei der DualenReihe üblich, als Taschenbuch im DINA4Format, erstmals mit zusätzlicher OnlineVersion und lässt sich über einen Zugangscode bequem auf das Tablet oder Smartphone runterladen und jederzeit abrufen. INHALT: Das Buch startet mit der allgemeinen Radiologie mit den Themen: physikalische Grundlagen, der Strahlenbiologie, Grundlagen des Strahlenschutzes, radiologische Verfahren, Strahlentherapie und Nuklearmedizin. Der zweite Hauptteil des Buches, die spezielle Radiologie, ist organspezifisch geordnet und behandelt die Themen: Thorax, Herz, Urogenitaltrakt und Retroperitoneum,
Skelett, Gefäßsystem/interventionelle Radiologie, Gastrointestinaltrakt, Leber, Pankreas, biliäres System und Milz, Mamma, ZNS, Wirbelsäule und Spinalkanal, Schädelbasis, Gesichtsschädel und Orbita, Hals und schließt mit bildgebenden Verfahren im Kindesalter. Typisch bei der Dualen Reihe ist das Kurzlehrbuch am Seitenrand, das einen zügigen Überblick ermöglicht und sich zur Prüfungsvorbereitung einfacher wiederholen lässt.

Dieses Lehrbuch ist strukturiert und didaktisch klar gegliedert aufgebaut. Über 1600 radiologische Bilder bzw. Abbildungen aus dem klinischen Alltag, multiple Schemazeichnungen, Tabellen und farbliche Markierungen tragen zur Veranschaulichung bei. Der Text beschränkt sich auf das Wesentliche und findet sich, wie oben bereits erwähnt, zusammengefasst als Randspalte. Darüber hinaus sind über vierzig klinische Fälle themenbezogen eingeflochten. Daher ist das Buch gerade für Studenten und Anfänger so attraktiv. Hilfreich sind die Gegenüberstellungen verschiedener bildgebender Verfahren (z. B. das ÖsophagusKarzinom in der CT und in der PETDarstellung oder entsprechende Endoskopie Befunde). Vor und Nachteile der jeweiligen Bildgebungen werden diskutiert. Auch bereits von der Schnittbilddiagnostik abgelöste und als obsolet geltende Verfahren sind der Vollständigkeit halber mit aufgeführt. PREISLEISTUNGSVERHÄLTNIS: Das PreisLeistungsverhältnis ist mit rund 80 Euro angemessen, vor allem wegen der zusätzlichen Nutzungsmöglichkeit der OnlineVersion. ADRESSATEN: Das Buch wurde für Jungassistenten der Radiologie oder Assistenzärzte in Weiterbildung anderer Fachrichtungen geschrieben, die im Dienst mit radiologischen Bildern konfrontiert werden und sich einen ersten Überblick über das Fach verschaffen möchten. Aber auch für Medizinstudenten in der Prüfungsvorbereitung kann es eine gute Basis bieten. Darüber hinaus ist es für interessierte MTRAs durchaus empfehlenswert.

\section{FAZIT}

Die Duale Reihe Radiologie bietet einen Überblick über die komplexen Themengebiete dieses Fachs. Dieses Buch versteht sich als Grundlage, um radiologische Bilder zu interpretieren und eine entsprechende Befunderstellung zu erlernen. Nicht nur für Medizinstudenten, sondern auch für Ärzte in der Weiterbildung ist es hilfreich und empfehlenswert.

Dr. Sonja Siepe, Detmold

\section{Messverfahren und Klassifikationen in der muskuloskelettalen Radiologie}

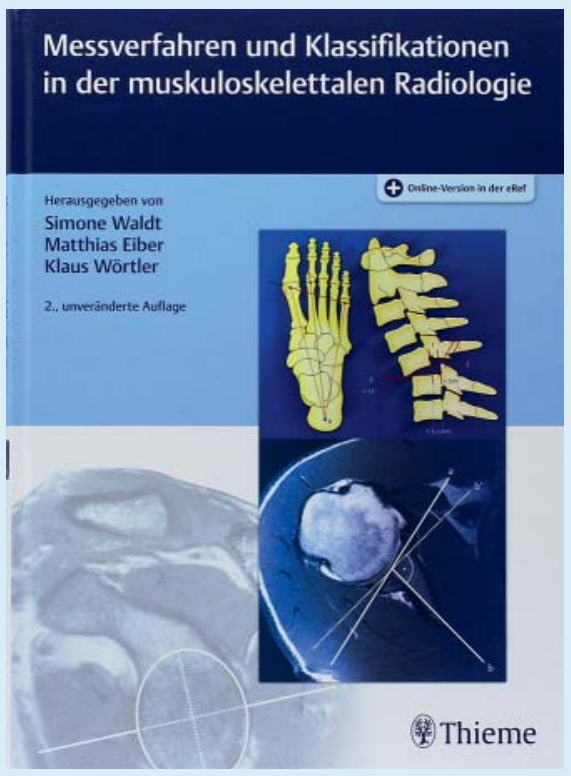

Simone Waldt, Matthias Eiber, Klaus Wörtler, 212 Seiten, 423 Abbildungen, 2. Auflage, Thieme, ISBN: 9783132415 133, 149,95€

Nach sechs Jahren gibt es eine Neuauflage des Klassikers und wie auch die 1. Auflage dient das Buch zum raschen und effektiven Nachschlagen der wichtigsten Messverfahren und Klassifikationen in der muskuloskelettalen Radiologie. Die klare übersichtliche Struktur und das ansprechende Layout wurden beibehalten und verfeinert. Der Aufbau orientiert sich weiterhin an anatomischen Regionen und Krankheitsbildern. Neben der Gliederung in die einzelnen Körperregionen des Bewegungsapparats gibt es weiterhin Kapitel zu Tumoren, Osteoporose, Gelenkknorpel, rheumatoider Arthritis, Beinachse, Muskelverletzung, Arthrose, Hamophilie und Skelettreifung. Ausgespart 
ist unverändert die Frakturlehre. In jedem der 17 Kapitel gibt es einen erklärenden Text, sehr übersichtliche Tabellen, überdurchschnittlich hochwertige Zeichnungen und Patientenaufnahmen, überwiegend Röntgenbilder mit farbigen Markierungen. Dies unterstützt nicht nur das Verständnis, sondern erhöht auch den Spaß am Lesen. Grau unterlegte Kästen erklären die klinische Relevanz der Messung oder Klassifikation. Die Klassifikationen sind sehr gut recherchiert und mit Literaturangaben versehen. Der Text ist gut verständlich und prägnant; mehr als 400 Schemabilder und radiologische Aufnahmen mit spezifischen Einzeichnungen illustrieren die beschriebenen Messungen und Klassifikationseinteilungen anschaulich. Neben der detaillierten Beschreibung wird der praktische Stellenwert der Methoden erläutert. Das Buch beschränkt sich auf die am häufigsten zur Anwendung kommenden Messungen und Klassifikationen in der muskuloskelettalen Radiologie; veraltete, obsolete Techniken werden entweder gar nicht oder nur mit speziellen Hinweisen erwähnt.
FAZIT

Auch die 2. Auflage ist eine praktisch orientierte, aktuelle Sammlung für eine qualitativ hochwertige Befundung - zum schnellen Nachschlagen der sich nur schwer zu merkenden Zahlen, Winkel, Messungen und Einteilungen.

Dr. Julia Kalinka-Grafe, Berlin

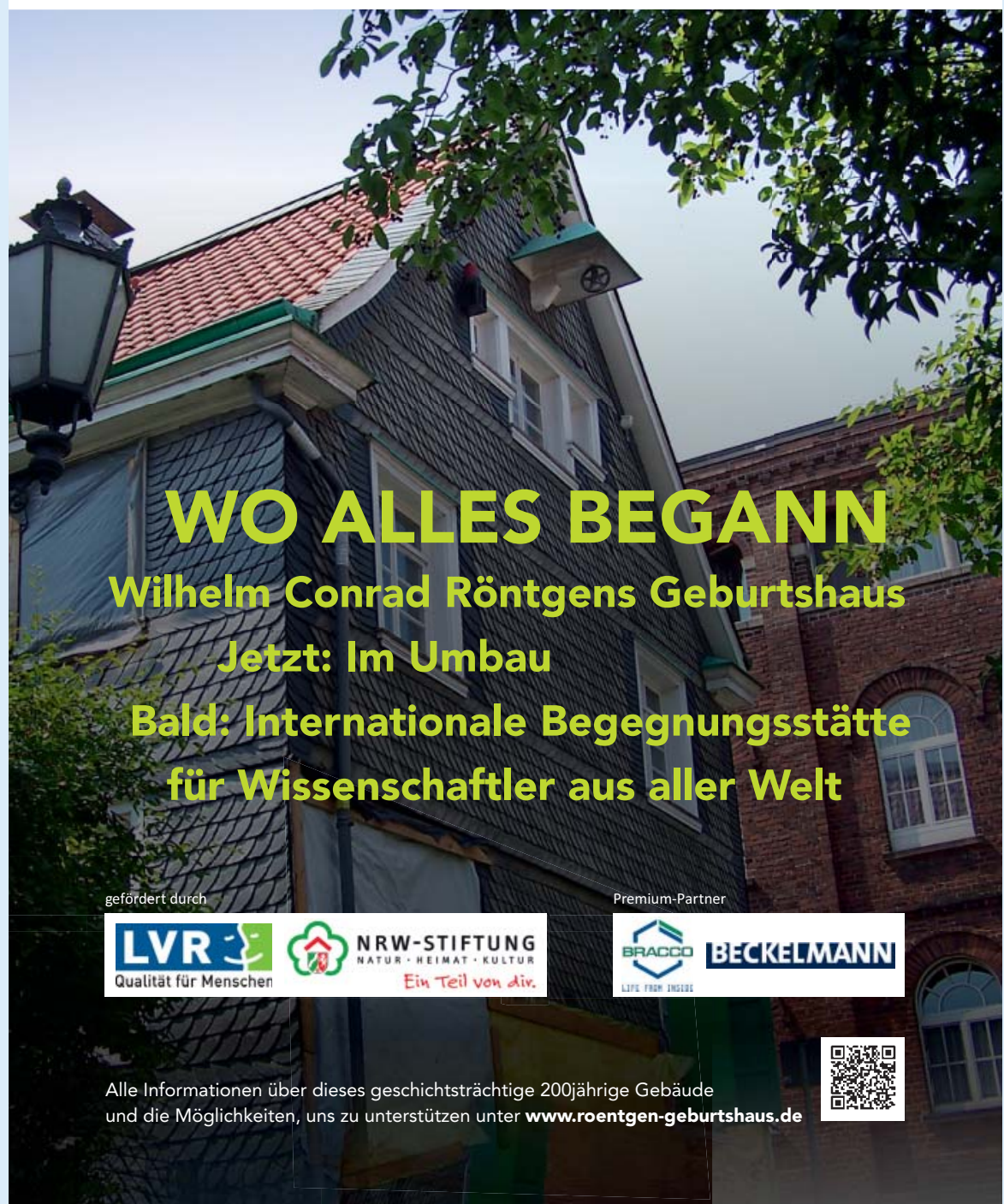

